\title{
Las drogas y la salud pública: ¿hacia dónde vamos?
}

\author{
María Elena Medina-Mora, Dra. en Psic, ${ }^{(1)}$ Tania Real, M en C, ${ }^{(1)}$ \\ Jorge Villatoro, Lic. en Psic, ${ }^{(2)}$ Guillermina Natera, M en Psic. ${ }^{(3)}$
}

\author{
Medina-Mora ME, Real T, Villatoro J, Natera G. \\ Las drogas y la salud pública: ¿hacia dónde vamos? \\ Salud Publica Mex 2013;55:67-73.
}

\section{Resumen}

El artículo presenta una propuesta para la atención del problema de las adicciones desde un enfoque de salud pública, define sus alcances y limitaciones frente a los retos del fenómeno, especialmente para la formulación de políticas; se suma a las propuestas que buscan integrar los temas sociales en la evaluación del resultado de las acciones y que proponen como meta última el bien de la persona y de las comunidades con una perspectiva de bienestar para la población. Describe el reto que enfrenta México en su papel como país productor, de tránsito y de consumo, analiza brevemente la evidencia sobre las políticas públicas y hace una serie de recomendaciones al respecto.

Palabras clave: abuso de sustancias; prevención y control; tratamiento; salud pública; políticas públicas
Medina-Mora ME, Real T, Villatoro J, Natera G.

Drugs and public health: where are we heading to? Salud Publica Mex 2013;55:67-73.

\section{Abstract}

This paper describes a plan for the attention of addictions from a public health perspective; it defines strengths of this perspective and its limitations to face the special challenges that the problem represents for social policies; adopts a wider perspective that includes the integration of health and social issues in measuring policy results having as a main aim the benefit of the person and of the communities with a perspective of the populations well being. It describes the challenge the country faces as producer, route of traffic and rates of consumption, analyzes briefly the evidence of public policies and makes a series of recommendations.

Key words: substance abuse; prevention and control; treatment; public health; public policies
$\mathrm{L}$ as drogas en sus diferentes manifestaciones han invadido nuestro entorno. Se trata de un fenómeno de múltiples facetas que incluye las etapas de producción, tráfico, distribución, consumo y dependencia; afectan a todos los grupos de la población y tienen implicaciones importantes para la salud pública de la nación. El problema requiere de enfoques novedosos que permitan enfrentar un problema complejo y cambiante. El objetivo de este ensayo es presentar una propuesta para la atención del problema de las adicciones desde un enfoque de salud pública y definir sus alcances y limitaciones frente a los retos que el fenómeno presenta, especialmente para la formulación de políticas.

Las drogas impactan en múltiples esferas, afectan el desarrollo económico y social, ${ }^{1}$ aumentan los costos de atención a la salud al asociarse con lesiones y con más

(I) Instituto Nacional de Psiquiatría Ramón de la Fuente. México, DF. México.

(2) Unidad de Encuestas, Instituto Nacional de Psiquiatría Ramón de la Fuente. México, DF. México.

(3) Investigaciones Epidemiológicas y Psicosociales, Instituto Nacional de Psiquiatría Ramón de la Fuente. México, DF. México.

Fecha de recibido: 24 de mayo de 2012 - Fecha de aceptado: 28 de agosto de 2012

Autor de correspondencia: María Elena Medina Mora. Doctora en Psicología Social, Instituto Nacional de Psiquiatría Ramón de la Fuente. Calzada México-Xochimilco I0I, I4370, México, DF. Correo electrónico: medinam@imp.edu.mx. 
de 60 enfermedades infecciosas (i.e., VIH, hepatitis B y C) y crónicas (cirrosis, cardiovasculares, cáncer, enfermedades mentales, etc.). ${ }^{2}$ Tienen una manifestación visible en diferentes formas de violencia que puede estar asociada con el crimen organizado, con actividades delictivas vinculadas con la adquisición de drogas en el mercado ilegal o inducidas por la intoxicación con sustancias psicoactivas. ${ }^{3,4}$

De acuerdo con Babor, ${ }^{5}$ existen tipologías de problemas que definen la situación de los diferentes países en cuanto al tráfico de drogas, el consumo interno y las políticas públicas. Así, por ejemplo, Estados Unidos define su problema como un asunto de justicia criminal, tiene muy altos niveles de consumo tanto ocasional como problemático, bajo nivel de tráfico, altos niveles de violencia y muy altos niveles de crimen atribuido a los usuarios de drogas. México, que lo define como un problema de seguridad nacional, tiene tasas más bajas de consumo ocasional y problemático, muy altos niveles de tráfico y violencia, pero el crimen que puede atribuirse a los usuarios es aún bajo.

Atender este complejo fenómeno implica incidir en la oferta y sus consecuencias, en la demanda y sus implicaciones y en el contexto, que incluye una cultura que valida violencia como mecanismo para resolver conflictos. El problema no puede entenderse si no se integran los componentes de oferta y demanda, tiene un carácter único, obliga a aprender de su historia y a buscar soluciones propias.

\section{La perspectiva de la salud pública}

Definir el fenómeno de las drogas desde una perspectiva de salud pública permite reconocer diferencias entre las drogas y sus riesgos; se aleja de conceptualizaciones que ven a las drogas como fin último, con el decomiso y la detención de personas como la meta, en cambio ve a las sustancias en su interacción con las personas que las usan o tienen potencial para hacerlo, que viven en contextos con mayor o menor riesgo y que son más o menos vulnerables a la experimentación y al paso del uso a la dependencia por una combinación de factores heredados y adquiridos. Se define el problema como una enfermedad y por tanto no se ve en el encarcelamiento de los enfermos la solución de los problemas.

Esta visión permite, también, analizar el consumo de sustancias y sus variaciones en frecuencia y cantidad, tanto como factor de riesgo para sufrir lesiones o centrarse en el estudio de la dependencia como una enfermedad. La perspectiva de salud pública reconoce variaciones entre las sustancias y sus efectos, y asimismo favorece dictar políticas acordes; identifica diferencias entre hombres y mujeres en sus necesidades de atención, y facilita in- tegrar el papel de la cultura y del contexto en la forma en que el problema se manifiesta.

A pesar de estos importantes alcances de la perspectiva de salud pública, los problemas sociales no pueden conceptualizarse sólo sobre la base de los indicadores de salud; ${ }^{5}$ por ejemplo, un mismo homicidio que se registra en los indicadores de mortalidad puede implicar la muerte de un traficante, un policía, un comprador adicto y a lo mejor distribuidor que no ha pagado su deuda o una persona ajena que iba de paso. De la misma manera, la negligencia que sufre un niño cuando su madre es adicta afecta su calidad de vida y lo pone en riesgo de enfermar, pero sólo puede cuantificarse si a los tradicionales indicadores de morbimortalidad se añaden indicadores de calidad de vida o bienestar.

Todas las políticas tienen consecuencias no deseadas que deben considerarse frente a los beneficios que representa cada acción. Ejemplos de consecuencias no deseadas son la violencia por la lucha de mercados o el aumento en la adicción a narcóticos como resultado de la política de ampliación de su disponibilidad para la atención de los enfermos, práctica que crea mercados y la oportunidad de la desviación. Conceptualizar el problema desde una perspectiva de salud pública, en la que el foco de atención sean la salud y el bienestar social, permite evaluar riesgos y ventajas de las diferentes medidas y por tanto orientar la acción minimizando costos.

\section{¿Cómo se manifiesta el problema en México?}

México cuenta con un sistema avanzado de recuperación de información útil para la toma de decisiones. ${ }^{6}$ Merced a estos estudios se ha podido conocer que, como en todo el mundo, el consumo de sustancias presenta periodos de crecimiento y disminución. ${ }^{1}$ A México lo distingue su situación geográfica, vecino del mercado de consumo más grande del mundo y vía de paso para la cocaína que se produce en la región andina con el objetivo principal de llegar a Estados Unidos y en medida decreciente hacia Europa. México produce opio y se ha convertido después de Afganistán (74\% de la producción potencial mundial de opio) en el segundo productor de opio en el mundo para el mercado ilegal con $5 \%$ de la producción mundial, que abastece principalmente el mercado norteamericano. ${ }^{7}$ Esta sustancia, cuyo consumo local se había mantenido en la frontera norte del país, se ha extendido a otras localidades. ${ }^{8,9}$ Estos antecedentes ponen el escenario para un incremento de hepatitis B, $\mathrm{C}$ y de VIH.

El cultivo de hoja de coca se concentra en la región andina-Colombia, Perú y Bolivia-. México se ha convertido en la principal ruta de salida con destino -en 
su mayor parte- al mercado americano. Se estima que 90\% de la cocaína decomisada en Estados Unidos pasó por la frontera con México. ${ }^{7}$ El uso de esta sustancia se incrementó considerablemente en nuestro país sobre todo desde finales de la década de los noventa; en el transcurso de diez años, de 1988 a 1998, aumenta 4.3 veces (de 0.33 a $1.45 \%$ ); para 2008 había 7.87 usuarios de cocaína por cada uno que la usaba en 1988; los grupos más vulnerables consumen crack, que es más adictivo, presenta mayor asociación con violencia y un mayor reto para el tratamiento. Las diferentes fuentes de información ${ }^{9-12}$ muestran, hasta 2011, un decremento en los índices de consumo de cocaína.

México, después de 2005, se convierte en el principal abastecedor de metanfetaminas en el mercado de Estados Unidos cuando ese país controla los medicamentos que contienen los precursores para la producción de estas drogas. En lo que se refiere a la demanda, su uso empieza a documentarse en México a partir de 1994;12 entre 2002 y 2008 la incidencia acumulada aumentó seis veces, de 0.08 a $0.5 \%$, en la población rural y urbana entre 12 y 65 años. ${ }^{9}$

En contraste, la mariguana se produce en todo el mundo y en casi todas las latitudes. El mayor decomiso ocurre en América del Norte, principalmente en México (1 $658 \mathrm{tm}$ ) y en Estados Unidos (1 $447 \mathrm{tm}) .^{7}$ Actualmente, la mariguana es la droga de mayor consumo en la población en México ( $80 \%$ del total de consumo de drogas) y en la mayor parte del mundo (entre 76 y $83 \%$ ); ${ }^{5}$ es también la droga cuyo uso presenta el más alto índice de crecimiento. ${ }^{8-10}$

En cuanto a los inhalables, grupo heterogéneo de sustancias contenidas en productos industriales y del hogar que incluyen: solventes (tolueno, pegamentos, gasolina), aerosoles (pinturas, desodorantes, cocina), gases (gas de encendedor, extinguidores) y nitritos (limpiadores de cabezas de video, desodorantes para habitaciones), su consumo ha representado una preocupación importante para las autoridades sanitarias del país, habiendo, sin embargo, mostrado periodos de crecimiento y decremento.,10 Son las drogas de inicio más temprano y las más prevalentes hasta los 15 años; después de esta edad, la mariguana es más frecuente. El consumo de inhalables en la población general mostró un incremento de 2002 ( $0.53 \%$ de la población informó haberse intoxicado con estos productos alguna vez en su vida) a 2008 (cuando el informe al respecto fue de $0.83 \%$ ). ${ }^{8}$ Los inhalables siguen siendo las drogas de preferencia de los niños y adolescentes en situación de calle.

En las mujeres, ${ }^{13}$ el fenómeno de consumo de cualquier droga es más reciente. La Encuesta Nacional de Adicciones de 2008 mostró mayor índice de consumo en las adolescentes de 12 a 17 años que en las de 18 a 34; mientras que en los hombres la incidencia acumulada es mayor entre el grupo de 18 a 34 años. ${ }^{8}$ Una variable que influyó de manera importante en estas diferencias es la exposición a la oportunidad, ${ }^{8}$ cuando ésta se controla, las diferencias entre hombres y mujeres desaparecen. Diversos estudios muestran que en los hombres hay más asociación con conducta antisocial y en las mujeres, con la depresión. ${ }^{14}$ Los familiares de los enfermos y en particular las mujeres, quienes con más frecuencia están a cargo del cuidado de los enfermos, presentan también mayor riesgo de enfermedad y en particular de presentar depresión. ${ }^{15}$

La adolescencia es una etapa de especial riesgo, cuya evidencia es que $65 \%$ de los usuarios de drogas se inician antes de los 17 años y la media de edad de inicio de quienes usaron drogas y desarrollaron dependencia es también a los 17 años, en tanto que quienes usaron y no desarrollaron dependencia iniciaron el consumo dos años más tarde. ${ }^{8}$ El promedio de inicio para la dependencia al alcohol es más tardía y ocurre a los 25 años. El alcohol representa el principal problema de salud pública del país, se estima que el consumo per cápita de bebidas formales es de 4.5 litros para la población mayor de 15 años y de 1.4 litros adicionales si se considera el alcohol que no paga impuestos. ${ }^{16,17} \mathrm{Su}$ abuso se asocia con índices elevados de accidentes y lesiones por violencia. ${ }^{18}$ México presenta altos índices de problemas derivados de intoxicación aguda asociados con la ingesta de grandes cantidades de alcohol por ocasión de consumo y elevadas tasas de trastornos asociados con su uso crónico. Se suma la contribución del consumo de bebidas alcohólicas a la carga global de enfermedad en México, ${ }^{19}$ pues deriva en altos niveles de mortalidad por cirrosis y otras enfermedades. ${ }^{20}$

La enfermedad mental tiene una edad de inicio temprana, las encuestas de epidemiología psiquiátrica realizadas en México muestran que 50\% de los trastornos mentales se inicia antes de los 21 años. ${ }^{14}$ El inicio temprano se ha asociado con un mayor riesgo de dependencia a drogas cuando los adolescentes se encuentran con ellas, de manera que cuando anteceden problemas de ansiedad o depresión, el riesgo de consumir drogas y desarrollar dependencia a ellas se incrementa entre 3 y 10 veces. ${ }^{21}$

Existen múltiples factores sociales que aumentan la probabilidad de extensión de este problema; entre los más importantes se encuentra el desempleo juvenil y la falta de oferta educativa, que sientan bases para la venta y uso de drogas, así como para la migración internacional, que ha jugado un papel importante en la extensión del problema a escenarios rurales. ${ }^{22}$

México presenta niveles más bajos de consumo de sustancias que los encontrados en el Cono Sur, similares 
a los que se observan en la región andina y considerablemente más bajos que los registrados en Estados Unidos y Canadá. ${ }^{23}$

\section{El debate internacional en torno a las políticas públicas}

El debate allende las fronteras mexicanas es amplio y complejo, se trata de un campo regulado internacionalmente en el que la ideología desempeña un papel importante. El mayor debate gira en torno a la legalización o la prohibición, pero carece de claridad en sus objetivos y definiciones; por ejemplo, ¿se habla de un libre mercado de drogas?, ¿se trata de la mariguana o de todas las drogas?, de despenalización (manteniendo la prohibición y el registro de antecedentes penales, pero incluyendo medidas como ofrecer tratamiento en lugar de cárcel $u$ otras que se apliquen durante el proceso y que busquen reducir la severidad de las penas a imponer), también se habla de descriminalización (manteniendo la prohibición pero estableciendo solamente penas administrativas en lugar de la cárcel), de regulación de mercados (como el de medicamentos psicotrópicos). ${ }^{5}$ Cada una de estas opciones tiene implicaciones diferentes, por lo que cualquier propuesta debe de partir de una definición clara de conceptos.

El debate también plantea la disyuntiva de legalizar la mariguana o todas las drogas como un mecanismo para abatir los beneficios económicos que la prohibición representa para el crimen organizado. En este sentido es recomendable analizar por separado ambos temas: drogas y crimen organizado, drogas y salud pública. Es evidente que las drogas implican recursos muy importantes para el crimen organizado, sin embargo el incremento de la disponibilidad también conlleva importantes riesgos para la salud pública, que hasta ahora no ha podido enfrentar los retos que el libre mercado del alcohol implica para el exceso de mortalidad y días vividos sin salud, pérdida de productividad laboral, violencia, entre otros impactos. En este sentido habría que considerar que las drogas no son el único mecanismo de obtención de recursos económicos para el crimen organizado, los obtiene también de la extorsión, falsificación, secuestro, entre otros delitos. Por otra parte, la prohibición también trae consecuencias no intencionadas que incluyen la violencia. Entre estas dos posiciones de todo o nada existen mecanismos intermedios $^{5}$ que deberán ser objeto de análisis. Si se opta por liberalizar los controles para disminuir los costos de la política de restricción, habrá un incremento en el consumo. Esta medida deberá ser acompañada, por tanto, de los recursos económicos que permitan al sector salud hacer frente al problema con mejores y más amplias medidas de prevención y tratamiento.
En lo que se refiere al tema de la prevención, no todos los esfuerzos de prevención han sido efectivos, ${ }^{24}$ de ahí que sea necesario apoyar aquellos programas que han mostrado resultados. Los programas deben adecuarse a la población objetivo, no todos los individuos tienen los mismos problemas ni las mismas necesidades; varían los antecedentes del individuo (factores heredados y de desarrollo), el tipo de sustancias que se consumen, su vía de administración y el contexto en que ocurre el consumo. Se ha encontrado evidencia de impacto en la reducción del consumo por algunos programas preventivos. ${ }^{5,25}$ En este sentido, Caulkins ${ }^{26}$ ha documentado cómo, aún con cambios pequeños, las intervenciones universales son costo-efectivas, dado el gran volumen de población que impactan.

El debate en torno al alcohol no es profundo aunque la evidencia sobre los beneficios de diferentes mecanismos de control sea amplia,; ${ }^{16}$ destaca el limitar la cantidad de alcohol circulante y el consumo en adolescentes, en mujeres embarazadas, en personas enfermas y en ocasiones de riesgo, como al conducir automóviles, así como la información acerca de los niveles de consumo de alcohol que representan el menor riesgo para la salud. Todas ellas, medidas que son efectivas cuando se acompañan de normas orientadas a proteger la salud y si se busca su cumplimiento; un ejemplo es normar sobre los límites de alcohol en sangre permitidos al manejar un auto, efectuar pruebas de aliento a los conductores, con probabilidad razonable de que el conductor que ha violado la norma sea detectado, y establecer sanciones que se cumplan. Otras medidas que han probado efectividad son el consejo médico, las intervenciones breves y el tratamiento. A pesar de que no hay controversia sobre las medidas mencionadas en cuanto a su impacto sobre la salud, ésta se da porque se afectan intereses de la industria productora y de servicios, que ven disminuidas sus ganancias cuando se disminuye el consumo.

Por el contrario, el debate sobre la importancia del tratamiento desapareció cuando se obtuvo la evidencia suficiente para definir los trastornos por abuso de sustancias como enfermedades del cerebro, modeladas por el medio ambiente y susceptibles de tratamiento, con el mismo nivel de éxito que el que se observa en el tratamiento de otras enfermedades crónicas como la diabetes. ${ }^{27}$ Debe considerarse que la dependencia a sustancias psicoactivas toma tiempo en desarrollarse, asimismo, su tratamiento exitoso es prolongado y como en el caso de cualquier otra enfermedad crónica, se esperan periodos de descompensación, lo que obliga a que el tratamiento esté disponible cuando el paciente lo necesita. ${ }^{27}$ Por tratarse de enfermedades crónicas asociadas con periodos de descompensación, se ha propuesto adoptar criterios 
de evaluación de éxito en el tratamiento basados en la reducción de recaídas, en la disminución de su gravedad y en el impacto en la calidad de vida de la población, no exclusivamente en la abstinencia.

\section{Recomendaciones}

I. Adoptar un enfoque de salud pública para la definición de políticas públicas.

Con base en la revisión efectuada, se recomienda adoptar un enfoque de salud pública, integrando indicadores sociales en la evaluación del impacto de las políticas, en donde el bienestar del individuo y de la comunidad sea el fin último.

II. Fortalecer la investigación epidemiológica y la inversión en ciencia y tecnología.

Dado el dinamismo del fenómeno se recomiendan encuestas más frecuentes; realizar la investigación que permita aumentar nuestra capacidad de entender la violencia y su relación con el consumo de drogas, y hacer una mejor integración y difusión de la información. También debe apoyarse la investigación científica básica y aplicada, que lleve a nuevos y mejores modelos para atender el problema. De especial relevancia son los estudios de traslación de la información a modelos de intervención probados en condiciones reales, en las comunidades, incluyendo el estudio de las barreras estructurales, personales y de la cultura que limitan la aplicación de los modelos probados.

III. Abordar el problema con un enfoque integrado de reducción de la oferta y de la demanda.

Las acciones de salud pública deben integrarse en un marco más extenso; se requiere un enfoque integrado más que balanceado entre combate a la oferta y a la demanda, y fortalecer las acciones de reducción de ésta última. Se propone una autoridad nacional central y local en cada uno de los estados con responsabilidad sobre las políticas de reducción de la oferta, de la demanda y el desarrollo social sustentable, que permita consolidar consensos, asegurar apoyo de las autoridades, financiar y evaluar los proyectos y promover comunicación estrecha entre los diferentes actores. Es importante lograr la integración de la atención de las adicciones, y no sólo de sus consecuencias (cirrosis, lesiones, $\mathrm{VIH}$, etc.), en el quehacer de las instituciones, en particular en el Sistema Nacional de Salud, y lograr la incorporación y el apoyo a las organizaciones de la sociedad civil. Una política integrada debe considerar al alcohol, a las drogas médicas y a las drogas ilegales.
IV. Apostar más a la prevención.

Se propone un enfoque amplio, basado en la evidencia, que incluya la prevención tanto dirigida a toda la población como focalizada en los grupos más vulnerables, incluyendo a las familias de los enfermos; apoyar los programas que han mostrado resultados como los orientados al desarrollo de habilidades.

La prevención de daños asociados con el abuso de alcohol requiere voluntad política, las medidas probadas incluyen: la reducción de la disponibilidad a través del incremento de precios vía impuestos, medida que debe venir acompañada de control del alcohol informal; la disminución de la densidad de establecimientos, el control de aquellos que operan sin licencia o incumplen con la normatividad, el control de los horarios de venta, entre otras.

Las medidas disuasivas con impacto sobre la reducción de mortalidad por lesiones incluyen políticas de venta a personas intoxicadas en los establecimientos, los dispositivos de prueba de aliento en conductores y que implican límites legales para conducir, retiro de licencia a personas que conducen intoxicadas reiteradamente. Asimismo, fomentar la responsabilidad de los padres de familia sobre la conducta de sus hijos.

Es importante reconocer las necesidades diferentes de hombres y mujeres, y desarrollar programas con enfoque de género. De igual forma, dado que las adiciones inician en la infancia y se reconoce que la edad de más riesgo es la adolescencia, deben apoyarse medidas que limiten el acceso y uso durante este periodo. Estas estrategias deben formar parte de programas integrales de prevención orientados a mantener estilos de vida saludables y al desarrollo de competencias sociales, así como de otros factores de protección que hagan a los jóvenes resilientes y les permitan enfrentar los riesgos, que asimismo lleguen a la familia, al contexto de pares y a la comunidad.

El importante papel que la enfermedad mental en la infancia y adolescencia temprana desempeña en el riesgo de desarrollar dependencia, cuando los adolecentes con un trastorno mental no tratado se encuentran con las drogas, obliga a considerar el tratamiento de la enfermedad mental, especialmente en estas etapas de la vida, como una poderosa estrategia de prevención. Dada la diferencia en la edad de aparición, la ventana de oportunidad es de siete años. ${ }^{21}$ 
V. Invertir en tratamiento e integrar su atención en el Sistema Nacional de Salud.

Se busca una cobertura universal de tratamiento para las personas con trastornos por abuso de sustancias; se debe reconocer que la desintoxicación es sólo la primera etapa de la intervención y que sin medidas de tratamiento posteriores no es una medida efectiva; incluir terapia de sustitución de narcóticos, cuando el adicto acepta este tipo de tratamiento. De manera similar, al reconocer las diferencias individuales entre los enfermos se enfatiza la importancia de que el tratamiento pueda adaptarse a las necesidades de cada persona. Se debe incluir el tratamiento de la comorbilidad psiquiátrica y de otras complicaciones médicas, así como la atención de los problemas sociales (legales, maritales, rezago educativo, falta de habilidades laborales, etc.).

La familia juega un papel muy importante en el apoyo de la recuperación del enfermo, por lo que debe integrarse al tratamiento; no obstante, dado que estos trastornos la afectan, la familia debe tener acceso a tratamiento para atender sus propias necesidades, independientemente de si el adicto acepta la intervención o no.

Estas propuestas son factibles si el tratamiento se integra en el Sistema Nacional de Salud, se cuenta con un sistema eficiente de referencia de pacientes a diferentes modalidades de intervención y se hacen sinergias con diversas instituciones (v.gr., educativas, laborales, de prevención de $\mathrm{VIH}$, etc.) que permitan poner al alcance de la persona con dependencia todos los recursos que favorezcan una mejor calidad de vida. Es recomendable establecer alianzas con los grupos de la sociedad civil que ofrecen programas de ayuda mutua, la cual ha sido una medida útil para aumentar la probabilidad de abstinencia.

Se recomiendan programas en los que colaboren autoridades de salud y de procuración de justicia para facilitar la canalización a tratamiento y el monitoreo de adictos que han cometido delitos bajo efectos de las sustancias o con el fin de procurárselas, y prevenir que cometan nuevos delitos. Las personas con adicciones que están en las cárceles deben tratarse con modelos eficaces que incluyan la continuación del tratamiento después de la liberación, a fin de evitar que las personas con dependencia entren y salgan de la institución con este trastorno y por tanto vuelvan a delinquir.

Se recomienda incluir estrategias de reducción de daños como parte de un programa integral de prevención (por ejemplo, las medidas de detección de alcohol en aliento en automovilistas junto con programas de educación) y de tratamiento (por ejemplo, el intercambio de jeringas usadas junto con oferta de tratamiento para los enfermos que usan drogas por vía de la inyección), a fin de reducir el riesgo de VIH; también son recomendables las terapias de sustitución con metadona $\mathrm{u}$ otras drogas cuando son el tratamiento indicado para los enfermos y es una opción aceptada por ellos.

VI. Establecer sinergias con otras dependencias que permitan acciones coordinadas y su inserción en las políticas de desarrollo social.

Un programa de salud pública tendrá mejores resultados si se inserta en un programa global de gobierno que busque el desarrollo. Se propone pasar de un enfoque inicial propuesto por Naciones Unidas, que se limitaba buscar el desarrollo alternativo para las comunidades productoras mediante una combinación de incentivos y de disuasión, a uno más amplio que buscaría consolidar el desarrollo de estas comunidades rurales y las urbanas en donde se producen y distribuyen drogas, como parte de la estrategias de combate a la pobreza y de mejora de la seguridad mediante alternativas de educación y empleo, teniendo como meta la disminución de inequidades e incrementar el desarrollo social del país.

Declaración de conflicto de interés: los autores declararon no tener conflicto de interés.

\section{Referencias}

I. Ghodse, H. International drug control into the $2 \mathrm{I}^{\text {st }}$ century. Londres: Ashgate/University of London, 2008:242.

2. Single E, Rehm J, Robson L, Truong MV. The relative risk and etiologic fractions of different causes of death and disease attributable to alcohol, tobacco and illicit drugs use in Canada. CMAJ 2000; I62(I2): I669-1675. 3. Del Olmo R. La conexión criminalidad violenta/drogas ilícitas: Una mirada desde la criminología. En: Hopenhayn M, comp. La grieta de las drogas. Desintegración social y políticas en América Latina. Santiago de Chile: Naciones Unidas/CEPAL:83-88.

4. Goldstein, $P$. The drugs-violence nexus: $A$ tri-partite conceptual framework. Journal of Drug Issues 1985; I5(4):493-506.

5. Babor T, Caulkins J, Edwards G, Fischer B, Foxcroft D, Humphreys K et al. Drug policy and the public good. Londres: Oxford University Press, 2009. 6. Medina-Mora ME, Cravioto P, Ortiz A, Kuri P, Villatoro J. México: Systems for the epidemiological diagnosis of drug abuse. Bulletin on Narcotics, 2003;LV(I-2):I05-II9.

7. United Nations Office on Drug and Crime. World Drug Report 2011 [monografía en internet]. United Nations Publications, 201 I [consultado 201 I octubre]. Disponible en: http://www.unodc.org/documents/wdr/ WDR_2010/World_Drug_Report_2010_lo-res.pdf.

8. Secretaría de Salud, Consejo Nacional contra las Adicciones, Instituto Nacional de Psiquiatría Ramón de la Fuente, Instituto Nacional de Salud 
Pública. Encuesta Nacional de Adicciones 2008. Resultados por entidad federativa. Distrito Federal [monografía en internet], México: 2009 [consultado 20II octubre]. Disponible en: www.inprfm.org.mx.

9. Secretaría de Salud, Subsecretaría de Prevención y Promoción de la Salud, Dirección General Adjunta de Epidemiología, Dirección de Investigación Operativa Epidemiológica, Sistema de Vigilancia Epidemiológica de las Adicciones (sISVEA). Informe 2009 [monografía en internet]. México: 2009 [consultado 201I enero 25]. Disponible en: http://www.dgepi.salud. gob.mx/20I0/PDFS/SISVEA/informes_sisvea_2009.pdf.

10. Villatoro J, Gaytán F, Moreno M, Gutiérrez ML, Olivia N, Bretón M et al. Consumo de alcohol, tabaco y otras drogas en la Ciudad de México. Medición 2009. México: Instituto Nacional de Psiquiatría Ramón de la Fuente Muñiz, 2010.

I I. Ortiz A, Martínez R, Meza D. Grupo interinstitucional para el desarrollo del sistema de reporte de información en drogas. Resultados de la aplicación de la cédula: Informe Individual sobre Consumo de Drogas. Tendencias en el área metropolitana 1987-2009. México: Instituto Nacional de Psiquiatría Ramón de la Fuente Muñiz, 2009. Disponible en: www. inprf.org.mx

12. Centros de Integración Juvenil (CIJ). Sistema institucional de información epidemiológica del consumo de drogas 2007. México: Centros de integración juvenil, dirección de investigación y enseñanza, 2007:210. 13. Medina-Mora ME, Villatoro J, Rafful C, Gutiérrez M, Moreno M, López MA et al. ¿Cómo son las mujeres que pasan la barrera social? Un análisis de mujeres usuarias de drogas y alcohol en México. En: Romero M, Medina-Mora ME, Rodríguez-Ajenjo C, eds. Mujeres y adicciones. México: CONADIC, 2011:73-I29.

14. Medina-Mora ME, Borges G, Lara C, Benjet C, Blanco J, Fleiz C et al. Prevalencia de trastornos mentales y uso de servicios: Resultados de la encuesta nacional de epidemiología psiquiátrica en México. Salud mental 2003:26(4): I- 16.

15. Natera G, Mora J, Tiburcio M, Medina P. An international perspective: Constructing intervention strategies for families in Mexico. Drugs: Education, Prevention and Policy 2010;17(I):193-202.

16. Medina-Mora ME, Robles R, Corina D, Real T. Evaluación de políticas públicas para el control del abuso de alcohol en México. México: Instituto Nacional de Psiquiatría Ramón de la Fuente Muñiz/Secretaría de Salud, 2009.
17. Medina-Mora ME, Villatoro J, Rodríguez CJ, Robles R. Consultores Internacionales SC. Alcohol no regulado en México. En: Ma. Elena Medina-Mora ME, Robles R, Cortina D, Real T, eds. Evaluación de políticas públicas para el control del abuso de alcohol en México 2009. México: Instituto Nacional de Psiquiatría, 2009.

I8. Borges G, Medina-Mora ME, Cherpitel C, Casanova L, Mondragón L, Romero M. Consumo de bebidas alcohólicas en pacientes de los servicios de urgencias de la ciudad de Pachuca, Hidalgo. Salud Pública de México 1999;4I(I):3.

19. González E, Gutiérrez C, Stevens G, Barraza M, Porras R, Caravalho $\mathrm{N}$ et al. Priority setting for health interventions in Mexico's system of social protection in health. The Lancet 2006;368(9547):1608-16I8. 20. Monteiro ME. Alcohol and public health in the Americas. A case for action. Washington, DC: Organización Panamericana de la Salud/Organización Mundial de la Salud, 2007.

21. Medina-Mora ME, Rojas E, Borges G, Vázquez L, Fleiz C. Depression and substance abuse. En: Aguilar-Gaxiola SA, Gullotta TP, eds. Depression in Latinos. Nueva York: Springer Science \& Business Media, 2008:73-90.

22. Borges G, Medina-Mora ME, Orozco R, Fleiz C, Cherpitel C, Breslau J. The Mexican migration to the United States and substance use in northern Mexico. Addiction 2009;104(4):603-6II.

23. Medina-Mora ME, Real T, Robles R. Epidemiology of drug abuse: A global overview. En: Ghodse H, Herrman H, Maj M, Sartorius N, eds. Substance abuse disorders: Evidence and experience. Chichester: Wiley, $201 \mathrm{I}$. 24. Sloboda Z, Stephens RC, Stephens PC, Grey SF, Teasdale B, Hawthorne RD et al. The adolescent substance abuse prevention study: A randomized field trial of a universal substance abuse prevention program. Drug and Alcohol Dependence 2009; I, I02(I-3): I- I0.

25. Babor T, Caetano R, Casswell S, Edwards G, Giesbrecht N, Graham $\mathrm{K}$ et al. El alcohol: un producto de consumo no ordinario. Washington, DC: Organización Panamericana de la Salud, 2010.

26. Caulkins J, Liccardo R, Paddock S, Chiesa J. School-based drug prevention. What kind of drug use does it prevent? Santa Mónica: Drug Policy Research Center/Rand, 2002.

27. McLellan AT, McKay JR, Forman R, Cacciola J, Kemp J. Reconsidering the evaluation of addiction treatment: From retrospective follow-up to concurrent recovery monitoring. Addiction 2005; 100(4):447-458. 